\title{
REPRESENTATION OF FUNCTIONS ANALYTIC IN A MULTIPLY-CONNECTED DOMAIN
}

By Yûsaku KOMATU

1. We may and do use, as a canonical domain of multiplicity $n(>2)$, a concentric annular ring silt along concentric circular arcs. Let the boundary components of such a domain $D$, laid on $z$-plane, be

$$
\begin{aligned}
& C_{1}: \quad|z|=1 ; \quad C_{2}:|z|=Q(<1) ; \\
& C_{j}: \quad|z|=m_{j}, \quad \theta_{j} \leqq \arg z \leqq \theta_{j}+\gamma_{j} \\
& (3 \leqq j \leqq n) \text {, }
\end{aligned}
$$

and the interior and the exterior sides of the slits $C_{j} \quad(3 \leqq j . \leqq n)$ be

$$
\begin{aligned}
& C_{j}^{(i)}:|z|=m_{j}-0, \quad \theta_{j} \leqq \arg z \leqq \theta_{j}+\gamma_{j}, \\
& C_{j}^{(e)} . \quad|z|=m_{j}+0, \quad \theta_{j}+\gamma_{j} \geqq \arg z \geqq \theta_{j},
\end{aligned}
$$

respectively. The total boundary of $D$ be denoted by

$$
C=\sum_{j=1}^{n} C_{j} \text {. }
$$

Any function $U(z)$ regular barmonic in the domain $D$ and continuous on the closed domain $D+C$ is represented by Green's formula in the form

$$
U(z)=\frac{1}{2 \pi} \int_{C} U(\zeta) \frac{\partial g(\zeta, z)}{\partial \nu_{\zeta}} d s_{\zeta},
$$

$g(\zeta ; z)$ being, as usual, "Green function (with variable $\zeta$ ) of $D$ with singularity at $z, v_{3}$ and $s_{3}$ denot. ing inward normal and arc-Jength parameter at a boundary point $\zeta$.

If we denote the equation of the boundary $C$ by $z=\zeta(s)$ and the har monic measure of a part of $C$ from a fixed point to the point $\zeta(s)$ by $\omega(z, \zeta(s))$, then we have

$$
\begin{aligned}
\frac{1}{2 \pi} \frac{\partial g(\zeta, z)}{\partial \nu_{\zeta}} d s_{\zeta} & =\alpha \omega(z, \zeta(s)) \\
& \equiv \omega(z, d \zeta(s)) .
\end{aligned}
$$

But, we use here an another aggregation, namely the one corresponding to Herglotz type. Let $\Phi(z)$ be an analytic function one-valued and regular in $D$ and continuous on $D+C$. We denote by $G(\zeta, z)$ ax analytic function of $z$ whose real part coincides with $g(5, z)=G(5, z)$ being uniquely determined except an additive purely imaginary quantity depending possibly on $\zeta$ and possessing mult1valuedness due to perlodiclty modul1 with respect to the boundary components. We have then, by the formula mentioned above,

$$
\Phi(z)=\frac{1}{2 \pi} \int_{C} x \Phi(\zeta) \frac{\partial G(\zeta, z)}{\partial \nu_{\xi}} d s_{\zeta}+i c,
$$

$c$ being a real constant.

We now assume that $R \Phi(z)$ is of bounded variation along $C$. Then, so is also the function $\left(\zeta \in C_{j}\right)$

$$
\rho_{j}(\varphi)=\int^{\varphi} R \Phi(\zeta) d s_{\zeta} \quad(\varphi=\arg \zeta) ;
$$

in fact,

$$
\int_{C_{j}}\left|d \rho_{j}(\varphi)\right|=\int_{C_{j}}|R \Phi(\zeta)| d s_{\zeta} .
$$

In this case, we may write the expression as in the Herglotz typo which states

$$
\Phi(z)=\frac{1}{2 \pi} \sum_{j=1}^{n} \int_{C_{j}} \frac{\partial G(\zeta, z)}{\partial y_{\zeta}} d p_{j}(\varphi)+i c .
$$

Now, considering residue at point $z$ we have particularly

$$
\frac{1}{2 \pi} \int_{C} \frac{\partial G(\zeta, z)}{\partial \nu_{\zeta}} d s_{S}=1 \text {, }
$$

pna hence

$$
1=\frac{1}{2 \pi} \sum_{j=1}^{n} \int_{C_{j}} \frac{\partial G_{r}(\zeta, z)}{\partial \nu_{\zeta}} d \sigma_{j}(\varphi),
$$

where $\sigma_{j}(\varphi)$ is defined by

$$
\sigma_{j}(\varphi)=\left\{\begin{array}{ll}
\varphi & \text { on } C_{1}, \\
-Q \varphi & \text { on } C_{2} ; \\
m_{j}\left(\varphi-\theta_{j}\right) & \text { on } C_{j}^{(i)}, \\
-m_{j}\left(\varphi-\theta_{j}-\gamma_{j}\right) & \text { on } C_{j}^{(s)}
\end{array}\right\}(3 \leqq j \leq n) .
$$

The last equation shows that an add1tive purely imaginary constant ic cono talned in the general representation vanishes out for the particular func tion $\Phi(z) \equiv 1$ 。

2. Consider now an analytic function $f(z)$ one-valued and regular in $D$ and plecewise regular on $D+C$. 
The boundary points, finite in number, where the regularity of $f(z)$ is broken down, be

$$
z_{j \mu} \quad\left(\begin{array}{l}
\mu=1,2, \cdots, n_{j}, \\
j=1, n
\end{array}\right) .
$$

The existence of Iimits of $f^{\prime}(z)$ from both sides along $C$ will be assumed at each of such points.

We assume further that $f^{\prime}(z)$ vanishes nowhere on $D+C$ except at these exceptional points $z_{j \mu}$. The image of $D$ by mapping $w=f(z)$ then possesses, on Rlemann surface, a plecewise analytic boundary and the function $f(z)$ can be prolonged analytically over every boundary arc containing no exceptional point. Denoting generally by ' $\zeta$ any exceptional point, then the image-curve of $C$ possesses at $f\left({ }^{\prime} \zeta\right)$ an angular point. Denoting by $\alpha \pi$ the exterior angle at such an angular point with respect to the image-domain, the fump of axg $f^{\prime}(z)$ at ' $\zeta$ along $C$ is given by

$$
\arg \frac{f^{\prime}\left(\zeta_{+}\right)}{f^{\prime}\left(\zeta_{-}\right)}=(\alpha-1) \pi,
$$

$\zeta_{\text {s }}$ being infinitely adjacent pointa at both sides of $\zeta$.

The Image curve of $C$ will moroover have angular pointe, in general, also at th $\mathrm{f}$ imege-points of end-points of the sifts. If $f^{\prime}(z)$ is regular at such an end-point op and does not va nish there, then the exterior angle of the image-curve at $f(p)$ is 0 and the jump of arof $f^{\prime}(z)$ there vanishes out. But, if $p$ colncides with an exceptional point ' $\zeta$ for which the Image curve possesses an angular point with exterlor angle $\alpha \pi$, then the jump of axg $f^{\prime}(x)$ there becomes $\alpha \pi$ since arg $d z$ sumps there by $-\pi$.

Let ' $\zeta$ be an exceptional point coinciding with none of end-points of the slits and the corresponding angle $\alpha \pi$ be different from $2 \pi$. Inen

$$
\left(f(z)-f\left({ }^{\prime} \zeta\right)\right)^{1 /(2-\alpha)} \text { i.s regular }
$$
at a vicinity of ' $\zeta$ and has $' \zeta$ as a gimple pole: namely, the function $f(z)-f(\xi)$ is uniformized by a local parameter $(z-\zeta)^{2-\alpha}$. Therefore, $f(z)-f\left(\zeta^{\prime} \zeta\right)$, as a function of $(z-j)^{2-\alpha}$, possesses a simple pole at $/ \zeta$. In case $\alpha=2$, instead of $\left(z-z^{-} \zeta\right)^{2-\alpha}, 1_{r}\left(z-z^{\prime}\right)$ may be taken as a local uniformizing parameter. In any case, the function

$$
(z-\zeta)^{\alpha-1} f^{\prime}(z)
$$

is regular and non-vanishing around $/ \zeta$. If an exceptional polnt ' $\zeta$ colncldes with an end-point $p$ of a slit, then the power $2-\alpha$ in local parameter has tc be replaced by $(2-\alpha) / 2$.
In the following, we suppose none of exceptional points colncide with any one of end-points of the slits, 1. e., $\zeta \neq p$. But, if it happens $\zeta=0$. the only modification must be made, according to the fact stated just above, that $\alpha$ has to be replaced by $\alpha / 2+1$

Now, the function defined by

$$
\begin{aligned}
\Phi(z) & =z \frac{d}{d z} \lg \left(f^{\prime}(z) \prod_{j=1}^{n} \prod_{\mu=1}^{n}\left(z-z_{j \mu}\right)^{\alpha_{j \mu}-1}\right) \\
& =\frac{z f^{\prime \prime}(z)}{f^{\prime}(z)}+\sum_{j=1}^{n} \sum_{\mu=1}^{n} \frac{\left(\alpha_{j \mu}-1\right) z}{z-z_{j \mu}}
\end{aligned}
$$

is evidently one-valued and regular throughout $D+C$. Hence, it is expressible in the form

$$
\Phi(z)=\frac{1}{2 \pi} \sum_{j=1}^{n} \int_{C_{j}} \frac{\partial G(\zeta, z)}{\partial \nu_{\zeta}} d p_{j}(\varphi)+\iota c,
$$

c being a real constant and $\rho(\varphi)$ being a reai function of $\varphi=a x g$ given by

$$
P_{j}(\varphi)=\int^{\varphi} R \Phi(\zeta) d s_{\zeta} \quad \text { for } \quad \zeta \in C_{j} .
$$

The linear function $z /\left(z-z_{j \mu}\right)$ behlaves regularly everywhere except only at a simple pole $z_{j \mu}$ and 1 ts real part is identically equal to $1 / 2$ along $C_{j}$. It will be easily seen that a representation of the same type as given above for $\Phi(z)$ holds good also for such a function.(1) Hence, we obtain the following representation formula with respect to $f(z)$ :

$$
\frac{z f^{\prime \prime}(z)}{f^{\prime}(z)}=\frac{1}{2 \pi} \sum_{j=1}^{n} \int_{c_{j}} \frac{\partial G(\zeta, z)}{\partial v_{\zeta}} m_{j} \operatorname{darg} f^{\prime}(\zeta)+\alpha c^{*},
$$

$$
\begin{aligned}
& c^{*} \text { being a real. constant and } m_{1}=1 \text {, } \\
& m_{2}=Q .
\end{aligned}
$$

On the other hand, we have seen that, for particular case $f^{\prime}(z) \equiv z$, the corresponding representation reduces to

$$
L=\frac{1}{2 \pi} \sum_{j=1}^{n} \int_{C_{j}} \frac{\partial G(\zeta, z)}{\partial \nu_{\zeta}} m_{j} \text { darg } \zeta,
$$

an adilive constant vanishing out. Hence, remembering that the relation

$$
\begin{aligned}
\operatorname{darg} d f(\zeta) & =\alpha \arg \left(\zeta f^{\prime}(\zeta) i d \varphi\right) \\
& =\alpha \arg \left(\zeta f^{\prime}(\zeta)\right) \quad(\varphi=\arg \zeta)
\end{aligned}
$$


1s valid along $C$, we have

$$
\begin{aligned}
1 & +\frac{z f^{\prime \prime}(z)}{f^{\prime}(z)} \\
= & \frac{1}{2 \pi} \sum_{j=1}^{n} \int_{C_{j}} \frac{\partial G(\zeta, z)}{\partial \nu_{s}} m_{j} \operatorname{daxg} d f(\zeta)+\iota c^{*} .
\end{aligned}
$$

The real constant $c^{*}$ can be determined as follows. For any fixed point $z_{0}$ in $D$, we put

$$
L(z, \zeta)=\frac{1}{2 \pi} \int_{z_{0}}^{z} \frac{\partial G(\zeta, z)}{\partial \nu_{\zeta}} \frac{d z}{z} .
$$

This function has a periodicity modulus around each boundary component $C_{j}$. Hence, if, introducing the uniformizing parameter $\lg z$, we put

$$
M(\lg z, \zeta)=L(z, \zeta),
$$

then the difference

$$
M(\lg z+2 \pi i, \zeta)-M(\lg z, \zeta)
$$

remains constant, for fixed $\zeta$, along each $C_{f}, 1 . \theta_{0}, z$ is contained in this expression oniy apparently. Hence, we may put

$$
\begin{array}{r}
M_{j}(\zeta)=M(\lg z+2 \pi i, \zeta)-M(\lg z, \zeta) \\
\left(z \in C_{j}\right)
\end{array}
$$

Integrating the above obtained expression for $f^{\prime \prime}(z) / f^{\prime}(z)$ with respect to $z$, we get

$$
\lg \frac{f^{\prime}(z)}{f^{\prime}\left(z_{0}\right)}=\sum_{j=1}^{n} \int_{C_{j}} M(g z, \zeta) m_{j} \operatorname{daxg} f^{\prime}(\zeta)+i c^{*} \lg \frac{z}{z_{0}} .
$$

Now, $f^{\prime}(z)$ being one-valued, the lefthand member of the last relation increases by an integral multiple of $2 \pi i$ for substitution $\lg z \mid 1 g z+2 \pi i$. Accordingly, the real part of this increase calculated from the right-hand member must vanish. Hence we get

$$
2 \pi c^{*}=\sum_{j=1}^{n} m_{j} \int_{C_{j}} R M_{j}(\zeta) \alpha \operatorname{dxg} f^{\prime}(\zeta),
$$

which is the relation determining $c^{*}$. Since, in particular case $f^{\prime}(z) \equiv z$, the corresponding constant decomes 0 , we may write also

$$
c^{*}=\frac{1}{2 \pi} \sum_{j=1}^{n} m \int_{C_{j}} R M_{j}(\zeta) \operatorname{darg} d f(\zeta) .
$$

The constant $c^{*}$ having beon determined, we obtain the desired reprosentation formuia

$$
1+\frac{z f^{\prime \prime}(z)}{f^{\prime}(z)}
$$

$$
=\frac{1}{2 \pi} \sum_{j=1}^{m} m_{j} \int_{C_{j}}\left(\frac{\partial G(\zeta, z)}{\partial \nu_{\zeta}}+R M_{j}(\zeta)\right) \operatorname{darg} d f(\zeta),
$$

which, by integration, yields a re

3. As an application of the above general formula, we consider here the case where $w=f(z)$ maps the basic domain $D$ onto a domain bounded by $n$ rectilinear polygons. Then, the exceptional points $/ \zeta$ are the points corresponding to vertices of the imagecurve of $C$, anc $2 r g d f$ becomes a step function having fump with hight $(\alpha-1)$ re at each $/ \zeta$. Hence, the general for.. mula roduces here to a simple form with out integration sign which states

$$
\begin{aligned}
& 1+\frac{z f^{\prime \prime}(z)}{f^{\prime}(z)} \\
= & \frac{1}{2} \sum_{j=1}^{n} m \sum_{j=1}^{n_{j}}\left(\alpha_{j \mu^{2}}-1\right) \frac{\partial \xi\left(z_{\partial \mu}, z\right)}{\partial z_{\gamma \beta}}+\ldots c^{i}
\end{aligned}
$$

$c^{*}$ being given by

$$
c^{*}=\frac{1}{2} \sum_{j=1}^{n} m \sum_{\mu=1}^{x_{j}}\left(\alpha_{j \mu}-1\right) R M_{j}\left(\varkappa_{j \mu}\right) .
$$

The successive integration yielas then

$$
\begin{aligned}
& \lg \frac{z f^{\prime}(z)}{z_{0} f^{\prime}\left(z_{0}\right)} \\
= & \pi \sum_{j=1}^{n} m \sum_{j=1}^{n_{j}}\left(\alpha_{j \mu}-1\right) L\left(z, z_{j \mu}\right)+\omega c^{*} \lg _{j} \frac{z}{z_{0}}+A_{i},
\end{aligned}
$$

$A_{1}$ being an irtegration constants and

$$
\begin{aligned}
& \begin{aligned}
& f^{\prime}(z) \\
= & \left.A z^{i c^{*}-i} \exp \left(\pi \sum_{j=1}^{n} m \sum_{j=1}^{n}\left(\alpha_{j \mu}-\cdots\right) L i r, z_{j \mu}\right)\right)
\end{aligned}
\end{aligned}
$$

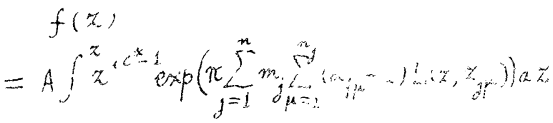

$A$ and $A^{\prime}$ denoting integration con stants which depend only on position and magnitude of the polygonal inage domain. The last formula may be we. garded as a generalization of SchwariChristof'el's one for simply-coniected case and of a formula for doubly-con nected case previously fiven by the present author. ( $(3)$ ( $(4)$

(*) Recelvod October 9, 1950 . 
(1) Gf. Y.Komatu, Darstellungen der in elnem Kre1sringe analytischen Funk tionen nobst den Anwendungen auf konforme Abblldung über Polygonalringgebiete. Jap. Journ. Math. 19 (1945), $203-215$.

(2) The corresponding formulae for simply - and doubly-connected cases have previously been given in Y.Komatu, Einige Darstellungen analytischer Funktionen und ihre Anwendungen auf kon-

forme Abblidung. Proc. Imp. Acad. Tokyo 20(1944),536-541 and in the paper cited (1), respectively.
(3) Joc. cit (1) and (2).

(4) For generalization of SchwarzChristoffel formula, see also Y.Komatu, Conformal mapping of polygonal domains, Journ. Math. Soc.Japan 2(1950), a preliminary note of which has been reported under the same titie in these Reports Nos. 3-4 (1949), 47-50。

Tokjo Institute of rechnology. 